\title{
The PROactive instruments to measure physical activity in patients with chronic obstructive pulmonary disease
}

\author{
Elena Gimeno-Santos ${ }^{1,2,3,4}$, Yogini Raste ${ }^{5}$, Heleen Demeyer ${ }^{6}$, Zafeiris Louvaris ${ }^{7}$, \\ Corina de Jong ${ }^{8}$, Roberto A. Rabinovich ${ }^{9}$, Nicholas S. Hopkinson ${ }^{5}$, \\ Michael I. Polkey ${ }^{5}$, Ioannis Vogiatzis ${ }^{7}$, Maggie Tabberer ${ }^{10}$, Fabienne Dobbels ${ }^{11}$, \\ Nathalie Ivanoff ${ }^{12}$, Willem I. de Boer ${ }^{13}$, Thys van der Molen ${ }^{8}$, Karoly Kulich ${ }^{14}$, \\ Ignasi Serra ${ }^{1,2,3}$, Xavier Basagaña ${ }^{1,2,3}$, Thierry Troosters ${ }^{6}$, Milo A. Puhan ${ }^{15}$, \\ Niklas Karlsson ${ }^{16}$ and Judith Garcia-Aymerich ${ }^{1,2,3}$ on behalf of the PROactive \\ consortium $^{17}$
}

\begin{abstract}
Affiliations: ${ }^{1}$ Centre for Research in Environmental Epidemiology (CREAL), Barcelona, Spain. ${ }^{2}$ Centro de Investigación Biomédica en Red de Epidemiología y Salud Pública (CIBERESP), Barcelona, Spain. ${ }^{3}$ Universitat Pompeu Fabra (UPF), Barcelona, Spain. ${ }^{4} F C S$ Blanquerna, Research Group in Physiotherapy (GReFis), Universitat Ramon Llull, Barcelona, Spain. ${ }^{5}$ National Institute for Health Research Respiratory Biomedical Research Unit, Royal Brompton and Harefield NHS Foundation Trust and Imperial College, London, UK. 'Department of Rehabilitation Sciences, Faculty of Kinesiology and Rehabilitation Sciences and Respiratory Division, University Hospital Leuven, Leuven, Belgium. ${ }^{7}$ Department of Critical Care Medicine and Pulmonary Services, Thorax Foundation, Athens, Greece. ${ }^{8}$ Department of General Practice, University Medical Center Groningen, University of Groningen, Groningen, The Netherlands. ${ }^{9}$ ELEGI Colt Laboratory, Centre for Inflammation Research, The Queen's Medical Research Institute, University of Edinburgh, Edinburgh, UK. ${ }^{10}$ Value Evidence and Outcomes, GlaxoSmithKline R\&D, Uxbridge, UK. ${ }^{11}$ Health Services and Nursing Research, Department of Public Health and Primary Care, University of Leuven, Leuven, Belgium. ${ }^{12}$ Almirall S.A., Barcelona, Spain. ${ }^{13}$ Lung Foundation Netherlands, Amersfoort, The Netherlands. ${ }^{14}$ PRO Expert, Novartis Pharma, Basel, Switzerland. ${ }^{15}$ Epidemiology, Biostatistics and Prevention Institute, University of Zurich, Zurich, Switzerland. ${ }^{16} \mathrm{Health}$ Economics and Outcomes Research, AstraZeneca R\&D, Mölndal, Sweden. ${ }^{17} \mathrm{~A}$ full list of the PROactive consortium members and their affiliations can be found in the Acknowledgements section.
\end{abstract}

Correspondence: Judith Garcia-Aymerich, Centre for Research in Environmental Epidemiology, Doctor Aiguader 88, 08003 Barcelona, Spain. E-mail: jgarciadacreal.cat

ABSTRACT No current patient-centred instrument captures all dimensions of physical activity in chronic obstructive pulmonary disease (COPD). Our objective was item reduction and initial validation of two instruments to measure physical activity in COPD.

Physical activity was assessed in a 6-week, randomised, two-way cross-over, multicentre study using PROactive draft questionnaires (daily and clinical visit versions) and two activity monitors. Item reduction followed an iterative process including classical and Rasch model analyses, and input from patients and clinical experts.

236 COPD patients from five European centres were included. Results indicated the concept of physical activity in COPD had two domains, labelled "amount" and "difficulty". After item reduction, the daily PROactive instrument comprised nine items and the clinical visit contained 14. Both demonstrated good model fit (person separation index $>0.7$ ). Confirmatory factor analysis supported the bidimensional structure. Both instruments had good internal consistency (Cronbach's $\alpha>0.8$ ), test-retest reliability (intraclass correlation coefficient $\geqslant 0.9)$ and exhibited moderate-to-high correlations $(r>0.6)$ with related constructs and very low correlations $(\mathrm{r}<0.3)$ with unrelated constructs, providing evidence for construct validity.

Daily and clinical visit "PROactive physical activity in COPD" instruments are hybrid tools combining a short patient-reported outcome questionnaire and two activity monitor variables which provide simple, valid and reliable measures of physical activity in COPD patients.

@ERSpublications

Both PROactive hybrid tools are simple, valid, and reliable measures of physical activity in COPD patients http://ow.ly/LJqP8 


\section{Introduction}

Physical activity is a key factor in determining health status, and its preventative role in chronic diseases is well accepted [1]. During the past decade, a substantial amount of research on physical activity in patients with chronic obstructive pulmonary disease (COPD) has been published, prompted initially by studies showing that low levels of physical activity are associated with poor prognosis [2, 3]. Research has consistently shown that patients with COPD have lower physical activity levels than their healthy peers [4-7] and many patients limit their physical activity to avoid symptoms [8]. A recent systematic review of the determinants and outcomes of physical activity in patients with COPD showed a consistent effect of regular physical activity in reducing COPD exacerbations and mortality [9]. Hence, physical activity constitutes an important outcome to clinicians and to patients with COPD.

Current trends in the assessment of physical activity focus on the measures of amount and frequency of activities using activity monitors [10]. However, it is unlikely that an activity monitor can fully capture patients' experience of physical activity. Indeed, qualitative research has shown that patients experience symptoms while being active, or have to adapt their lifestyle [11], features that will never be captured using activity monitors alone. Such a patient-centred concept can only be measured through a patient-reported outcome (PRO) questionnaire, which needs to be developed using data directly from patients with COPD [12]. Conversely, a PRO questionnaire alone will also not allow assessment of physical activity patterns or detection of small changes in physical activity on a day-to-day basis. Of note, the currently available PROs that aim to measure physical activity in COPD patients are not based on a conceptual framework [13] and their development does not follow best practice as set out in the guidelines published by the US Food and Drug Administration (FDA) [14]. In developing the PROactive tools we hypothesised that items generated from patient experiences in combination with an activity monitor would capture all relevant dimensions of physical activity in patients with COPD [11].

Within the framework of the European Union Innovative Medicines Initiative PROactive project (www. proactivecopd.com) our group first conducted qualitative research and drafted a conceptual framework to set out the concept of physical activity from patients' experience and to provide the necessary basis to generate an item pool for two PROs, one with a daily recall period and one with a 7-day recall period (daily and clinical visit versions, respectively) to measure the experience of physical activity in patients with COPD [11]. Our group has also evaluated the validity of six commercial physical activity monitors in patients with COPD $[15,16]$. The aim of the current study was 1) to identify a comprehensive set of nonredundant items and monitor outputs that can be used to measure physical activity in COPD patients and 2) to perform an initial validation of the two instruments.

\section{Methods}

Study design and sample

A 6-week, randomised, two-way cross-over, multicentre study was conducted in five European medical centres (fig. 1, further details in the online supplementary material). We recruited both stable and exacerbated patients with COPD defined by spirometry, to cover the whole range of physical activity. The study was advised and approved by the PROactive ethics and patient advisory boards, approved by the ethics committee at each centre and registered at www.clinicaltrials.gov (NCT01388218). Written consent was obtained from all patients.

\section{Assessments and measures}

Figure 1 shows the assessments and timing. In the first 2 -week study period patients were randomised to complete either the daily PROactive item pool, consisting of 30 questions asking patients to report their physical activity experience on a daily basis, or the clinical visit PROactive item pool, of 35 questions using a 7-day recall (see online supplementary material). Following a 2-week wash-out patients completed the other questionnaire during the second study period. Both item pools were developed previously using

Clinical trial: This study is registered at www.clinicaltrials.gov with identifier number NCT01388218.

This article has supplementary material available from www.erj.ersjournals.com

Support statement: This study was supported by the European Commission Innovative Medicines Initiative Joint Undertaking (IMI JU \#115011). There was no involvement of funding sources in study design; the collection, analysis, and interpretation of data; the writing of the report; nor the decision to submit the article for publication. This project was supported by the National Institute for Health Research Respiratory Biomedical Research Unit at the Royal Brompton and Harefield NHS Foundation Trust and Imperial College (London, UK) who part-fund Michael I. Polkey's salary. Funding information for this article has been deposited with FundRef.

Conflict of interest: Disclosures can be found alongside the online version of this article at erj.ersjournals.com 


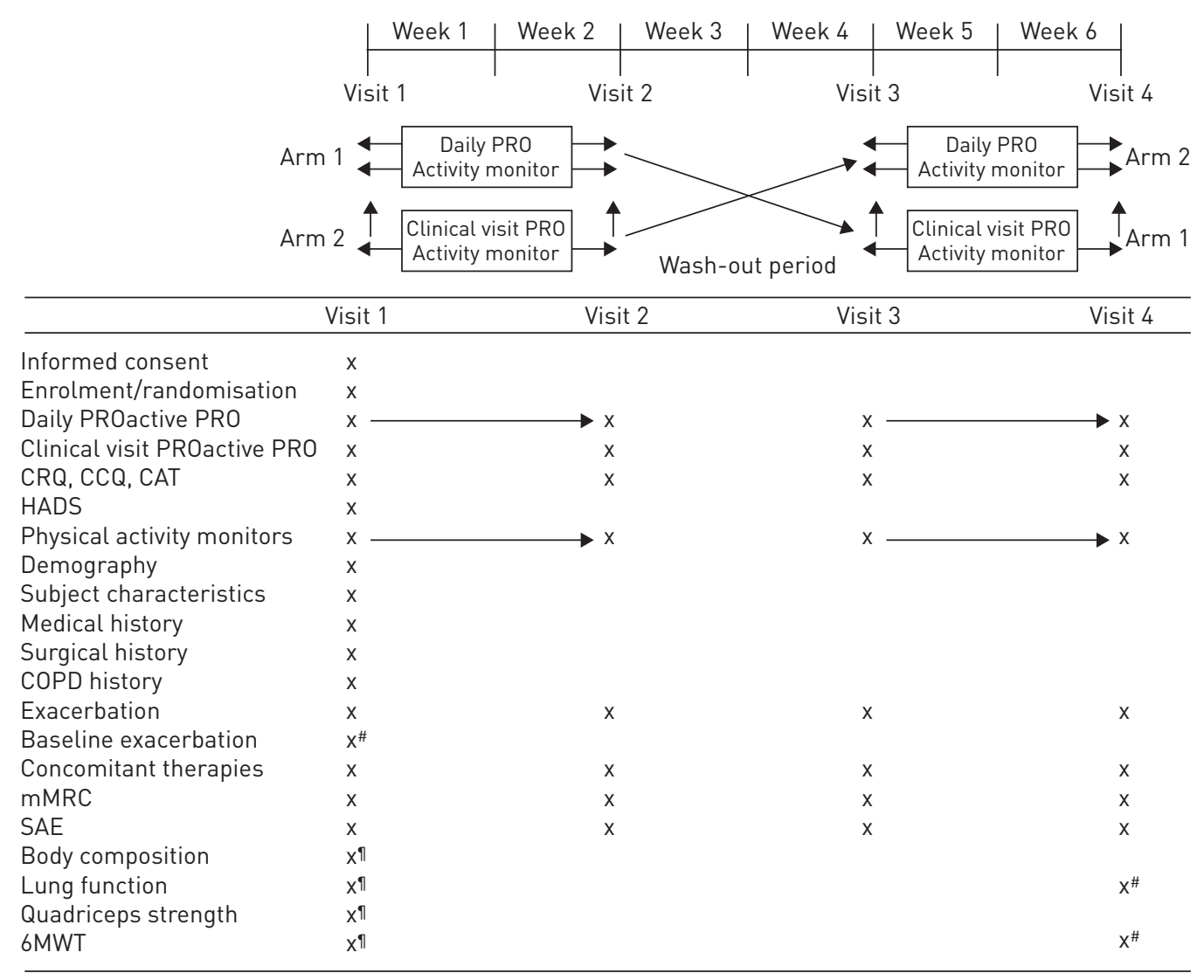

FIGURE 1 Study visits and assessments. PRO: patient-reported outcome; CRQ: chronic respiratory questionnaire; $C C Q$ : clinical chronic obstructive pulmonary disease questionnaire; CAT: chronic obstructive pulmonary disease assessment test; HADS: hospital anxiety and depression scale; COPD: chronic obstructive pulmonary disease; mMRC: modified Medical Research Council dyspnoea scale; SAE: serious adverse event; 6MWT: 6-min walk test. ${ }^{\#}$ : assessments could be split between visit 1 and visit 2 ; ${ }^{\text {? }}$ : in the exacerbated patients group only.

appropriate qualitative research methods and linguistic adaptation for each site [11] in line with current European Medicines Agency [17] and US FDA published standards [14].

During the study periods (fig. 1), patients wore two accelerometers previously identified during a systematic review [10] and further validated for COPD patients against the doubly labelled water method [15] and indirect calirometry [16] (Actigraph G3Tx; Actigraph, Pensacola, FL, USA and Dynaport MoveMonitor; McRoberts BV, The Hague, The Netherlands).

\section{Statistical analysis}

The analysis sets and sample size calculations, as well as the statistical analysis plan were defined a priori according to objectives (see online supplementary material).

The item reduction process was conducted separately for each PROactive instrument and followed a systematic iterative process (fig. 2 and online supplementary material). Items were flagged and considered for removal if they exceeded prespecified thresholds of acceptability [18-25]. We hypothesised a priori that PROs alone might not capture information on the amount of physical activity well, so both item pools were analysed alone and in combination with activity data from each monitor (analysed separately).

Confirmatory factor analysis was used to test the fit of the final PROactive Physical Activity in COPD (PPAC) instruments with the dimensionality identified during the item reduction process [20, 26]. Initial validation of the item-reduced PPAC instruments comprised reliability (internal consistency and test-retest reliability) and construct validity (convergent, known-groups and discriminant validity) [23, 27]. All analyses were performed separately for each instrument and dimension, in case the instruments exhibited more than one dimension. 


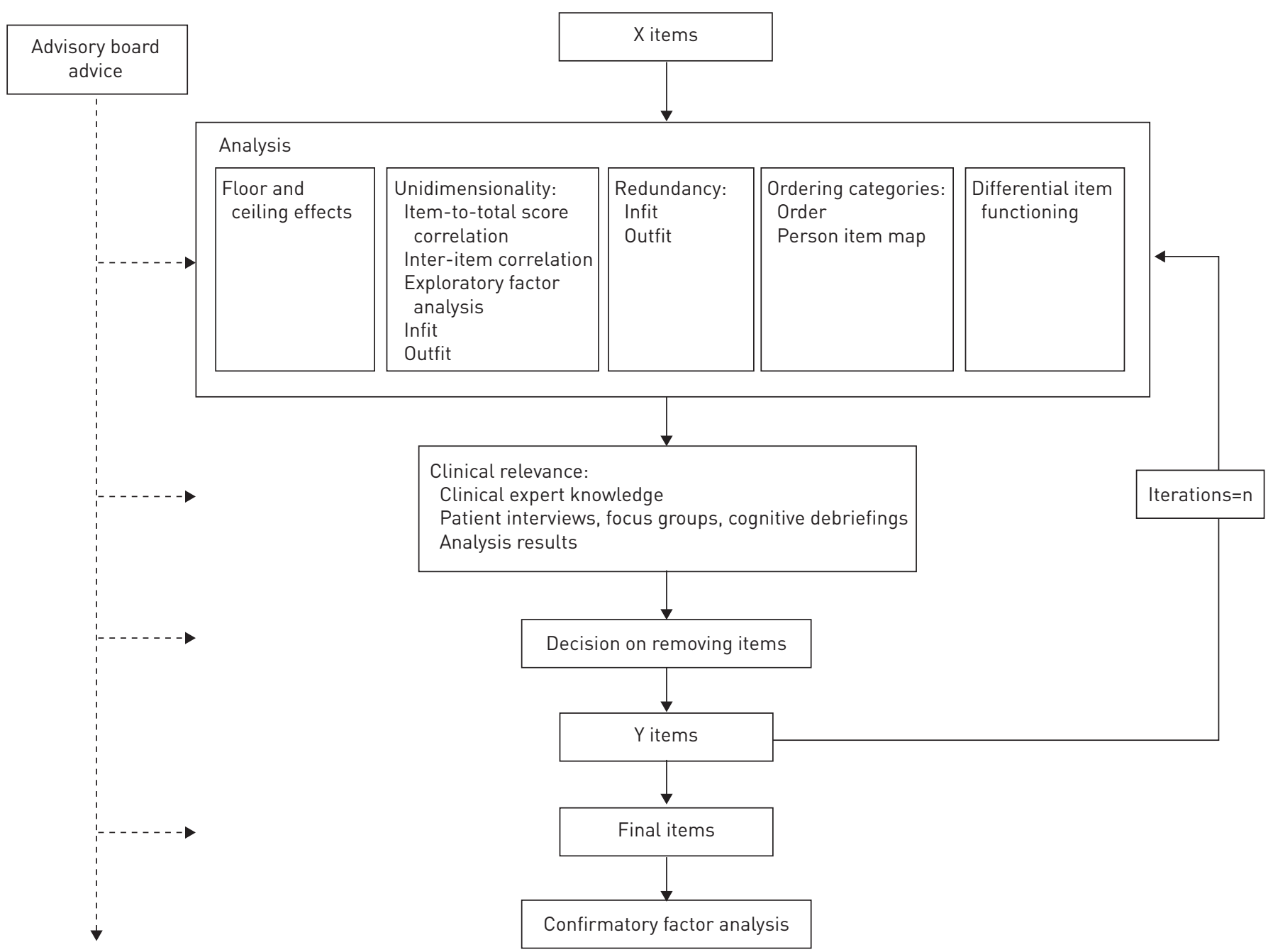

FIGURE 2 Flowchart of the iterative process for the item reduction process.

\section{Results}

236 patients with COPD were included in the study (fig. 1). Patients were mostly male (68\%), with mean \pm SD age of $67 \pm 8$ years, forced expiratory volume in $1 \mathrm{~s}$ of $57 \pm 21 \%$ and body mass index of $27 \pm 5 \mathrm{~kg} \cdot \mathrm{m}^{-2}$. Baseline characteristics are shown in table 1, and in online supplementary table E1 stratified by site. A total of 228 patients $(97 \%)$ had valid ( $\geqslant 3$ days with $\geqslant 10 \mathrm{~h}$ wearing time) data from activity monitors, showing good compliance and moderate levels of physical activity (online supplementary table E2).

\section{Item reduction}

The factor analysis, item-to-total and inter-item correlations, and infit and outfit statistics from the Rasch models suggested that both the daily and clinical visit versions of PPAC (D-PPAC and C-PPAC, respectively) had a bidimensional structure, with a clear distribution of items in two factors (online supplementary tables E3 and E4). Consequently, the process of item reduction was performed separately according to factors 1 "amount of physical activity" and 2 "difficulty with physical activity".

Tables 2 and 3 detail the reasons for item flagging for exclusion: due to floor or ceiling effects, not fitting unidimensionality, redundancy, not proper ordering, differential item functioning or less relevant for patients and/or experts. For the D-PPAC, a total of five iterations in factor 1 ("amount") allowed a reduction from nine to four items, including two variables from activity monitors (steps and vector magnitude unit (VMU)); and in factor 2 ("difficulty"), a total of 12 iterations allowed a reduction from 26 to five items. Full information on item removal is detailed in online supplementary table E5. The final version of the D-PPAC (see online supplement), described in figure 3, exhibited good performance and distribution of items, according to the person separation index (PSI) (0.83 in factor 1 with Dynaport, and 0.80 in factor 1 with Actigraph; 0.88 in factor 2) and the person-item map (fig. 4). Confirmatory factor 


\section{TABLE 1 Baseline demographic and clinical characteristics}

\section{Subjects $\mathbf{n}$}

Disease status at recruitment

Stable

Exacerbated

Age years

$67.4 \pm 8.4$

Male

Primary ethnicity: white

Living alone

Low socioeconomic status (NS-SEC IV-V) 39 (16)

Working status: employed $\quad 28(12)$

Current smoker

Smoking pack-years

BMI kg.m $\mathrm{m}^{-2}$

$27.0 \pm 5.5$

FFMI $\mathrm{kg} \cdot \mathrm{m}^{-2 \#}$

$18.1 \pm 3.0$

FEV $1 \%$ pred

$57.0 \pm 20.5$

FVC \% pred

$95.6 \pm 19.6$

FEV $/$ FVC $\%$

$46.9 \pm 13.2$

IC/TLC \%"

$34.3 \pm 9.9$

$\mathrm{RV} / \mathrm{TLC} \%^{\#}$

TLCo \% pred ${ }^{\#}$

$59.8 \pm 22.7$

\section{ATS/ERS stages ${ }^{\#}$}

I: mild (FEV $1 \geqslant 80 \%$ )

$34(15)$

II: moderate (FEV $1<80 \%$ and $\geqslant 50 \%$ )

$108(46)$

III: severe (FEV $1<50 \%$ and $\geqslant 30 \%)$

$71(30)$

IV: very severe (FEV1<30\%)

22 (9)

6MWD $\mathrm{m}^{\#}$

Quadriceps strength $\mathbf{k g}^{\#}$

Any admissions for COPD exacerbation ever

Coronary heart disease

Any cardiovascular disease or disorder

Musculoskeletal disorders

27 (11)

Dyspnoea (mMRC 0-4)

$1.7(1.1)$

CRQ dyspnoea\#

CRQ fatigue ${ }^{\#}$

$4.8(1.5)$

$4.3(1.3)$

$4.9(1.3)$

$5.3(1.3)$

CRQ mastery

$1.9(1.1)$

CCQ total ${ }^{\#}$

CAT $^{\#}$

AADS anxiety\#

$5.0(3.4)$

Data are presented as $\mathrm{n}, \mathrm{n}(\%)$ or mean \pm SD. NS-SEC: National Statistics Socio-economic Classification; BMI: body mass index; FFMI: fat-free mass index; FEV1: forced expiratory volume in $1 \mathrm{~s}$; FVC: forced vital capacity; IC: inspiratory capacity; TLC: total lung capacity; RV: residual volume: TLCo: transfer factor of the lung for carbon monoxide; ATS: American Thoracic Society; ERS: European Respiratory Society; 6MWD: 6-min walking distance; COPD: chronic obstructive pulmonary disease; mMRC: modified Medical Research Council dyspnoea scale; $C R Q$ : chronic respiratory questionnaire; CCQ: clinical COPD questionnaire; CAT: COPD assessment test; HADS: hospital anxiety and depression scale. ${ }^{\#}$ : some values are missing for certain variables (FFMI $n=9, I C / T L C n=15, R V / T L C n=14, T L C O n=8,6 M W D n=3$, quadriceps strength $n=3$, $C R Q$ dyspnoea $n=88, C R Q$ fatigue $n=3, C R Q$ emotional $n=3, C R Q$ mastery $n=3, C C Q n=1, C A T n=2, \operatorname{HADS}$ anxiety $n=1$, HADS depression $n=1$ ); ๆ : including coronary heart disease.

analysis supported the two-factor structure and exhibited appropriate goodness of fit (online supplementary table E6).

For the C-PPAC, a total of four iterations allowed reduction from nine to four items, including two variables from activity monitors for factor 1 . For factor 2, a total of 16 iterations allowed reduction from 31 to 10 items. The final version of the C-PPAC (see the online supplement), described in figure 3, also showed good performance and items distribution, as per the PSI ( 0.75 in factor 1 with Dynaport, 0.74 in factor 1 with Actigraph, and 0.93 in factor 2) and person-item map (fig. 4). Confirmatory factor analysis again supported the two factor structure and exhibited appropriate goodness of fit (table E6). 
TABLE 2 Performance of all items of the daily version of PROactive Physical Activity in COPD (chronic obstructive pulmonary disease) instrument (D-PPAC) and activity monitors during item reduction

Item flagged due to

\begin{tabular}{cccccc}
\hline $\begin{array}{c}\text { Floor/ceiling } \\
\text { effect }\end{array}$ & $\begin{array}{c}\text { Not fitting } \\
\text { unidimensionality }\end{array}$ & Redundancy & $\begin{array}{c}\text { Not proper } \\
\text { order }\end{array}$ & $\begin{array}{c}\text { Differential Item } \\
\text { Functioning }\end{array}$ & $\begin{array}{c}\text { Less important } \\
\text { according to } \\
\text { patients/experts }\end{array}$ \\
\hline
\end{tabular}

Factor 1 "Amount of physical activity"

Walking outside

Chores inside

Chores outside

Physical leisure

Intensity

Time in locomotion ${ }^{\#}$

Standing time ${ }^{\#}$

$\mathrm{VMU} \cdot \mathrm{min}^{-1 \#}$

Steps ${ }^{\#}$

MVPA

$\mathrm{VMU} \cdot \mathrm{min}^{-19}$

Steps

Factor 2 "Difficulty with physical activity"

Difficulty getting dressed

Difficulty carrying things

Difficulty climbing stairs

Difficulty showering

Difficulty taking a bath

Difficulty bending over

Difficulty washing yourself

Difficulty walking up a small slope

Difficulty when hurrying

Avoid activities

Breathless in general

Lack physical strength

Lack of strength in legs

Tired in general

Take breaks

Pace yourself

Prevented from doing activities

Breathless walking on level ground

Slow down while walking

Breathless climbing stairs

Time to recover

Spread activities

Stop doing activities

Puffs in addition

Consider lung problems when planning

Help from others

\begin{tabular}{|c|c|c|c|c|c|c|}
\hline & & & & & & $x$ \\
\hline & $x$ & & & $x$ & $x$ & \\
\hline & $x$ & & $x$ & & & $x$ \\
\hline & $x$ & & & & & \\
\hline & & $x$ & & & & \\
\hline & $x$ & & & $x$ & $x$ & \\
\hline & & & & & & $x$ \\
\hline & & & & & & $x$ \\
\hline & & $x$ & $x$ & & & \\
\hline & & & & & & $x$ \\
\hline & & & & & & $x$ \\
\hline & & & & & & $x$ \\
\hline$x$ & $x$ & & & & $x$ & \\
\hline$x$ & $x$ & & $x$ & & $x$ & \\
\hline$x$ & & & $x$ & & $x$ & \\
\hline$x$ & & & $x$ & & $x$ & \\
\hline$x$ & $x$ & & & $x$ & & \\
\hline$x$ & & $x$ & & & $x$ & \\
\hline$x$ & $x$ & & & & $x$ & \\
\hline$x$ & & & & & $x$ & \\
\hline & & & & & & $x$ \\
\hline & & & & & & $x$ \\
\hline & & $x$ & & & $x$ & \\
\hline & $x$ & & & & $x$ & \\
\hline & & & & & & $x$ \\
\hline & & & & & & $x$ \\
\hline & & $x$ & & & $x$ & \\
\hline$x$ & & $x$ & & & & \\
\hline & & $x$ & & & $x$ & \\
\hline & & $x$ & & & $x$ & \\
\hline & $x$ & & & & $x$ & \\
\hline & & $x$ & & & $x$ & \\
\hline$x$ & & & $x$ & & & \\
\hline$x$ & & $x$ & $x$ & & & \\
\hline$x$ & $x$ & & & & $x$ & \\
\hline$x$ & & $x$ & & & & \\
\hline$x$ & $x$ & $x$ & $x$ & & $x$ & \\
\hline
\end{tabular}

VMU: vector magnitude unit; MVPA: moderate-to-vigorous physical activity. " : data from Dynaport; ๆ : data from Actigraph. 
Item flagged due to

Floor/ceiling effect Not fitting unidimensionality Redundancy Not proper order Differential item functioning Less important according to patients/experts

\begin{tabular}{|c|c|c|c|c|c|c|c|}
\hline \multicolumn{8}{|l|}{ Factor 1 "Amount of physical activity" } \\
\hline Walking outside ${ }^{\#}$ & & & & $\mathrm{x}$ & & & $\mathrm{x}$ \\
\hline Chores inside & & $\mathrm{x}$ & & & $\mathrm{x}$ & $\mathrm{x}$ & \\
\hline Chores outside & & & & & & & $\mathrm{x}$ \\
\hline Physical leisure & & $\mathrm{x}$ & & $\mathrm{x}$ & & & \\
\hline Exercise to maintain or improve physical & & $\mathrm{x}$ & & $\mathrm{x}$ & & & \\
\hline Intensity" & & $\mathrm{x}$ & & & & & \\
\hline Time in locomotion" & & & $\mathrm{x}$ & & & & \\
\hline Standing time $e^{\pi}$ & & $\mathrm{x}$ & & & $\mathrm{x}$ & $\mathrm{x}$ & \\
\hline $\mathrm{VMU} \cdot \min ^{-19}$ & & & & & & & $\mathrm{x}$ \\
\hline Steps ${ }^{\pi}$ & & & & & & & $\mathrm{x}$ \\
\hline MVPA $^{\S}$ & & & & $\mathrm{x}$ & $\mathrm{x}$ & & \\
\hline $\mathrm{VMU} \cdot \mathrm{min}^{-1 \S}$ & & & & & & & $\mathrm{x}$ \\
\hline Steps ${ }^{\S}$ & & & & & & & $\mathrm{x}$ \\
\hline \multicolumn{8}{|l|}{ Factor 2 "Difficulty with physical activity" } \\
\hline Difficulty getting dressed & & & & & & & $\mathrm{x}$ \\
\hline Difficulty carrying objects & & $\mathrm{x}$ & & & $\mathrm{x}$ & $\mathrm{x}$ & \\
\hline Difficulty climbing stairs & & & & $\mathrm{x}$ & $\mathrm{x}$ & $\mathrm{x}$ & \\
\hline Difficulty showering & $\mathrm{x}$ & & & $x$ & $\mathrm{x}$ & $x$ & \\
\hline Difficulty bathing & $\mathrm{x}$ & & & $\mathrm{x}$ & & $\mathrm{x}$ & \\
\hline Difficulty bending over & $\mathrm{x}$ & $\mathrm{x}$ & & & $\mathrm{x}$ & $\mathrm{x}$ & \\
\hline Difficulty washing yourself & $\mathrm{x}$ & & & $\mathrm{x}$ & $\mathrm{x}$ & & \\
\hline Difficulty walking up small slope & & & & & & $\mathrm{x}$ & \\
\hline Difficulty getting out and about & & & & & & & $\mathrm{x}$ \\
\hline Difficulty when hurrying & & $\mathrm{x}$ & & & & $\mathrm{x}$ & \\
\hline Avoid activities & & & & & & & $\mathrm{x}$ \\
\hline Breathless in general & & & & & & & $\mathrm{x}$ \\
\hline Lack physical strength & & & & & & & $\mathrm{x}$ \\
\hline Lack of strength in legs & & $\mathrm{x}$ & & & & $\mathrm{x}$ & \\
\hline Tired in general & & & & & & & $\mathrm{x}$ \\
\hline Take breaks & & & & & & & $\mathrm{x}$ \\
\hline Pace yourself & & & $\mathrm{x}$ & & & $\mathrm{x}$ & \\
\hline Prevent doing activities & & & & $\mathrm{x}$ & $\mathrm{x}$ & & \\
\hline Cough during activities & & $\mathrm{x}$ & & & & $\mathrm{x}$ & \\
\hline Breathless walking on level ground & & & & & & & $\mathrm{x}$ \\
\hline Slow down while walking & & & $\mathrm{x}$ & & $\mathrm{x}$ & & \\
\hline Breathless climbing stairs & & & & & $\mathrm{x}$ & $\mathrm{x}$ & \\
\hline Time to recover & & & & & & & $\mathrm{x}$ \\
\hline Spread activities & & & & $\mathrm{x}$ & & & \\
\hline Stop doing activities & & & $\mathrm{x}$ & & & $\mathrm{x}$ & \\
\hline Puffs in addition & & $\mathrm{x}$ & & & & $\mathrm{x}$ & \\
\hline Consider lung problems when planning & & & & & & & $\mathrm{x}$ \\
\hline Help from others & $\mathrm{x}$ & & & & $\mathrm{x}$ & $\mathrm{x}$ & \\
\hline Use aids to facilitate activities & $\mathrm{x}$ & & & $\mathrm{x}$ & $\mathrm{x}$ & $\mathrm{x}$ & \\
\hline Overexert yourself during activities & & $\mathrm{x}$ & & & & $\mathrm{x}$ & \\
\hline
\end{tabular}

VMU: vector magnitude unit; MVPA: moderate-to-vigorous physical activity; ${ }^{\#}$ : item flagged because the original response categories did not show proper ordering; after collapsing the last two categories the item showed no ordering problems and therefore it was not removed. ${ }^{\text {ๆ: }}$ data from Dynaport; ${ }^{\S}$ : data from Actigraph. 


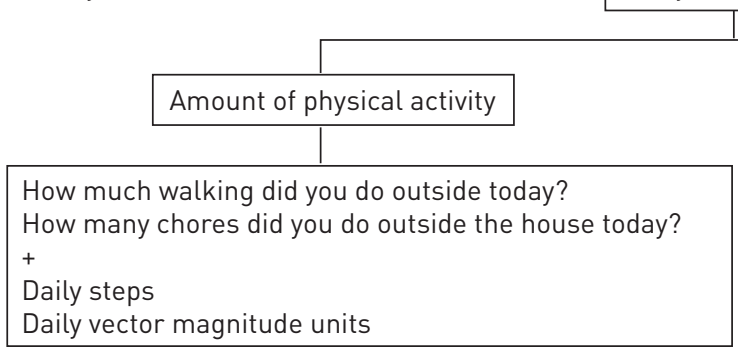

Difficulty during physical activity

How much difficulty did you have getting dressed today?

How often did you avoid doing activities because of your lung problems today?

How breathless were you in general during your activities today?

How tired were you in general during your activities today?

How often did you have to take breaks during your physical activities today?

b) Clinical visit version
\[ \begin{array}{l}\text { Amount of physical activity } \\ \text { In the past } 7 \text { days, how much walking did you do outside? } \\ \text { In the past } 7 \text { days, how many chores did you do outside } \\ + \\ \text { Weekly steps } \\ \text { Weekly vector magnitude units }\end{array} \]

FIGURE 3 Conceptual frameworks of a) the daily version of PROactive Physical Activity in COPD (chronic obstructive pulmonary disease) (D-PPAC) and b) the clinical visit version of PROactive Physical Activity in COPD (C-PPAC) instruments: final domains and items.

Several analyses were performed to select cut-off points for activity monitor variables to achieve optimal performance of the PPAC instruments. Because cut-offs were not identical between Actigraph and Dynaport, we determined agreement between the variables from the two monitors (complete methods are described in the online supplement) and observed $91 \%$ agreement for steps and $88 \%$ for VMU in the daily instrument, and $82 \%$ and $96 \%$ for steps and VMU, respectively, in the clinical visit version.

Complementary analyses using PRO data alone for factor 1 (i.e. excluding activity monitor data) showed that neither the D-PPAC nor the C-PPAC achieved optimal performance or discrimination. Specifically, all items exhibited low item-to-total correlation, some of them were flagged due to floor effect, redundancy, ordering problems and differential item functioning, and the PSI values decreased from 0.83 to 0.59 and from 0.75 to 0.62 in the daily and clinical visit versions, respectively, when the item reduction process did not include variables from the activity monitors (online supplementary table E7 and fig. E1). Taken together, the data show that the combination of PRO and monitor data resulted in better measurement properties than either PRO or monitor data alone.

Both PPAC instruments can be scored as a simple sum of the scores of the items (mostly from 0 to 4 ), with lower scores indicating poorer physical activity. The final PROactive scores exhibited no differences by sex, although they demonstrated lower values in the most severe COPD patients (online supplementary table E8).

\section{Reliability and construct validity}

Both PPAC instruments showed strong internal consistency and test-retest reliability (table 4 and online supplementary figs E2 and E3). In both PPAC instruments, factor 1 ("amount of physical activity") exhibited weak correlations with health-related quality of life and moderate correlations with dyspnoea and exercise capacity, while factor 2 ("difficulty with physical activity") scores showed moderate-to-strong 
a)
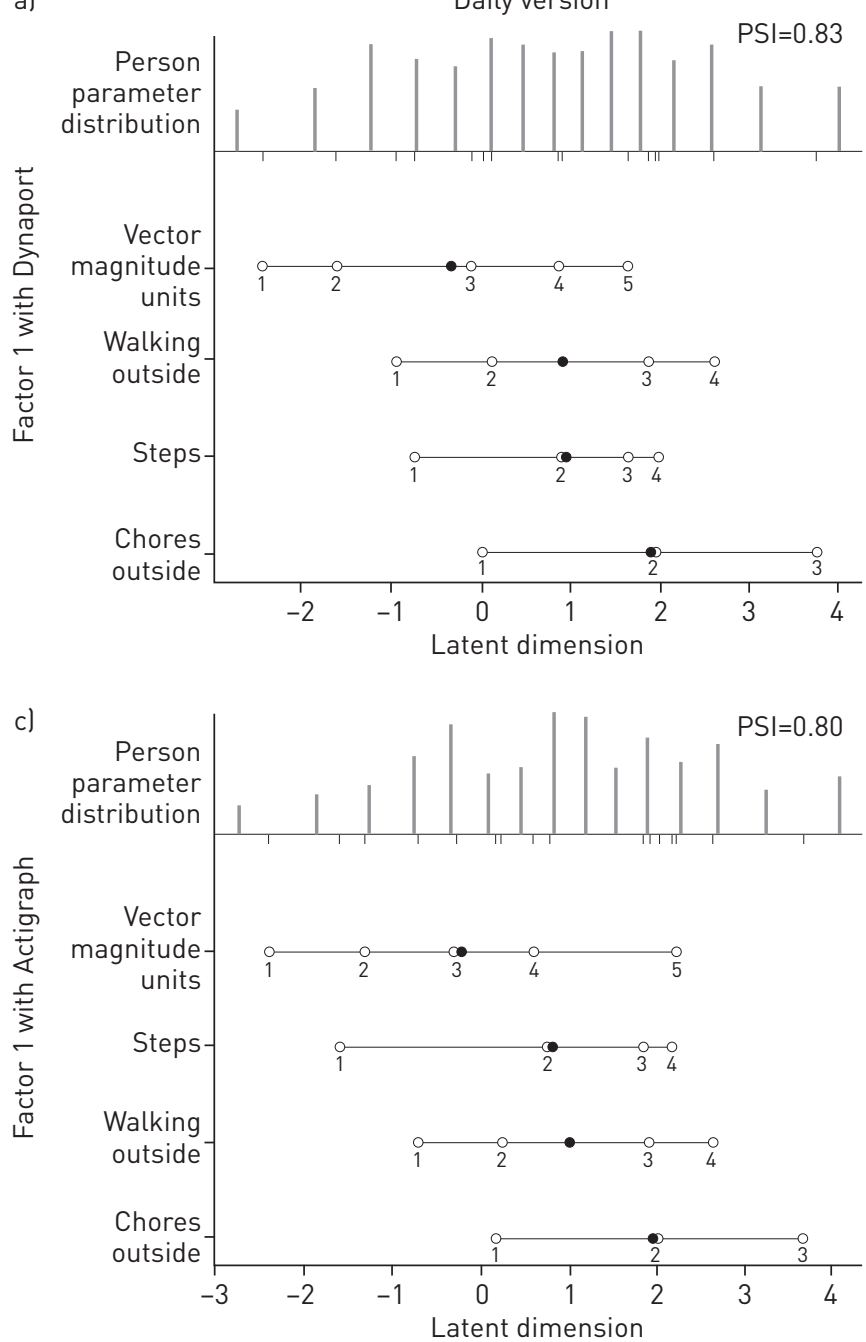

e)

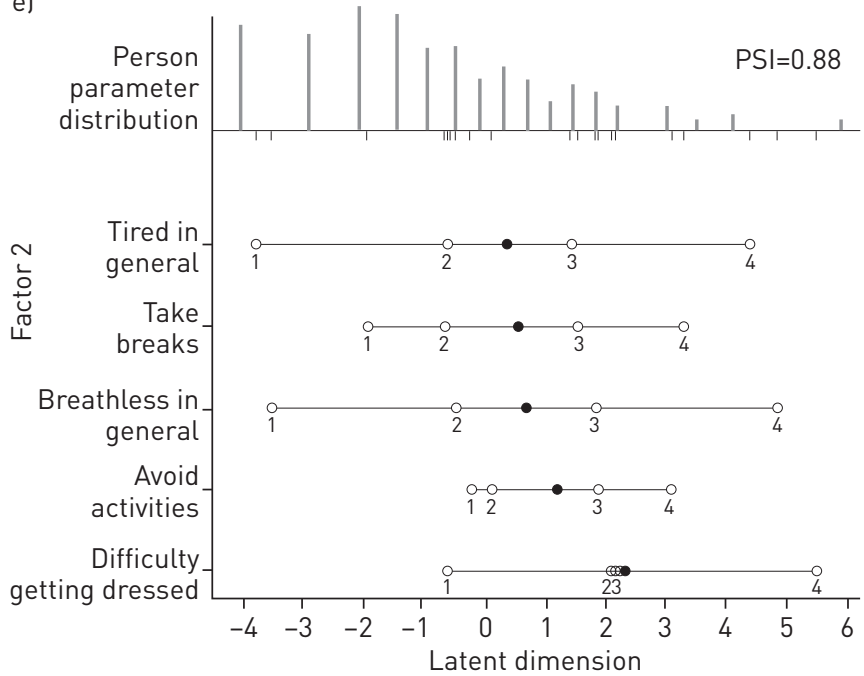

b)

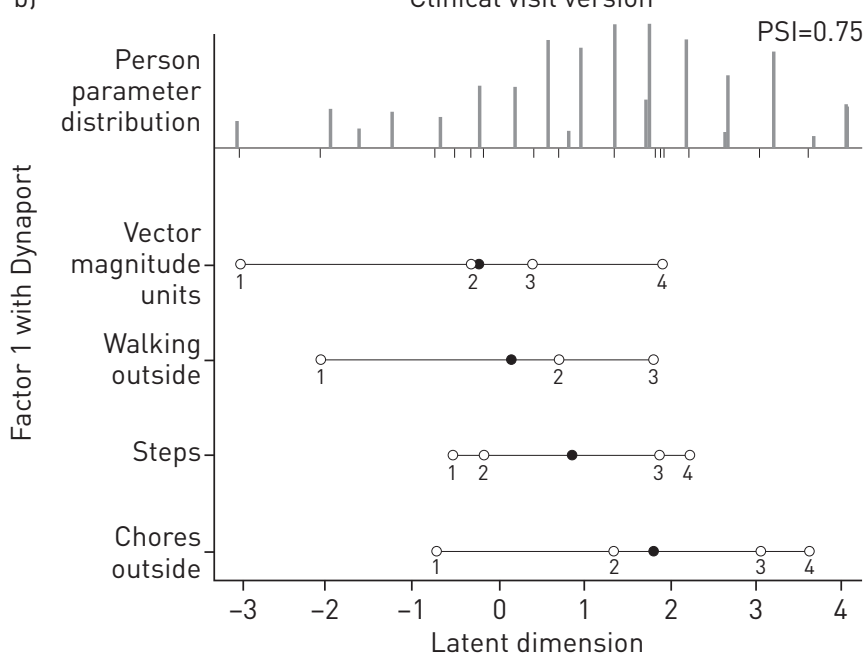

f)
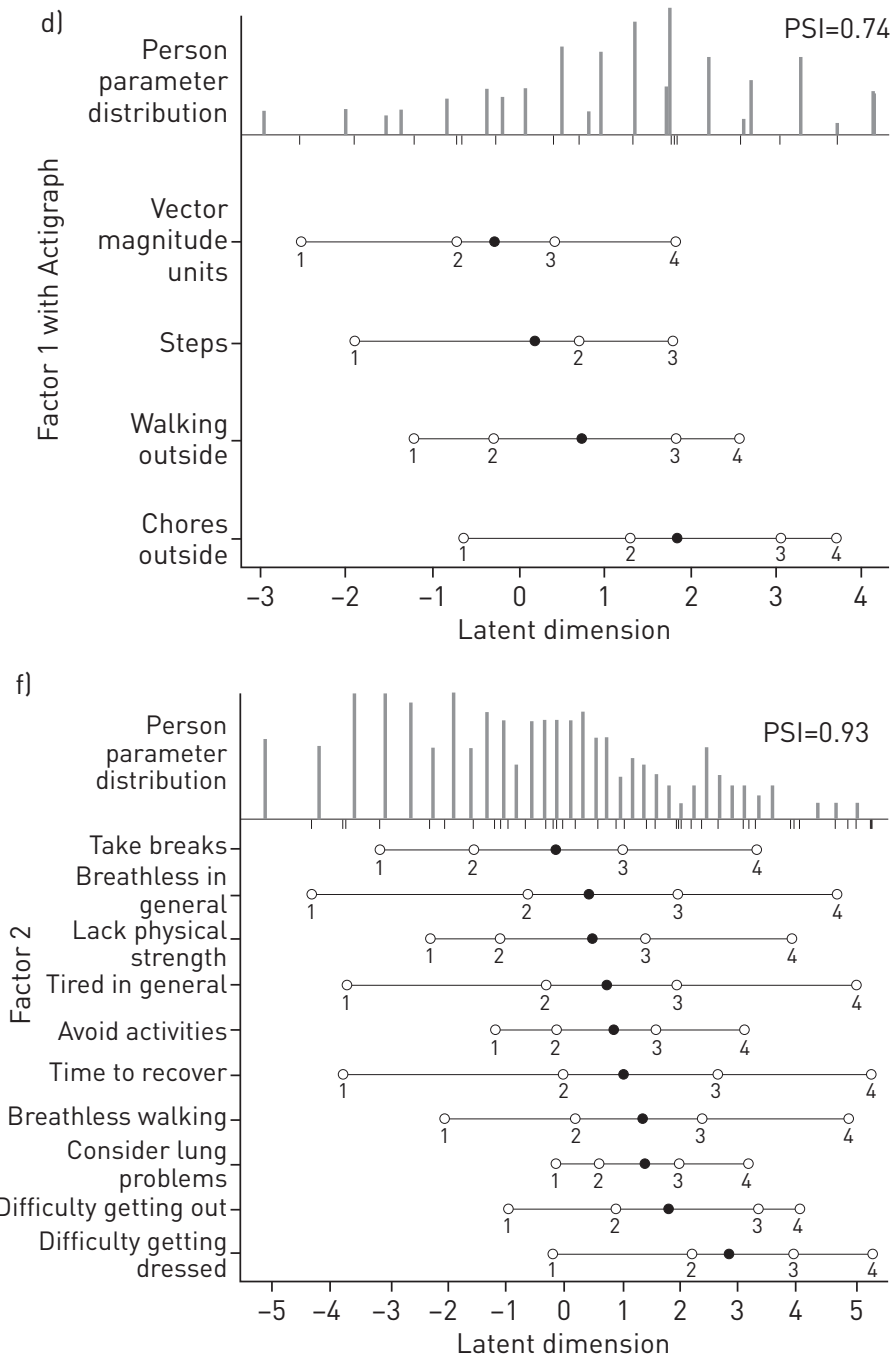

FIGURE 4 Person-item maps and overall fit of the model for factors 1 ("amount") and 2 ("difficulty") of the daily version of PROactive physical activity in COPD (chronic obstructive pulmonary disease) (D-PPAC) and the clinical visit version of the PROactive Physical Activity in COPD (C-PPAC) instruments. These plots indicate that the final items are well targeted to the patients and cover a wide range of the factor measured. Person separation index (PSI) values indicate that each factor is precise enough to differentiate between different patients' physical activity experiences. a) D-PPAC final items of factor 1 in combination with final variables of Dynaport; b) C-PPAC final items of factor 1 in combination with final variables of Dynaport; c) D-PPAC final items of factor 1 in combination with final variables of Actigraph; d) C-PPAC final items of factor 1 in combination with final variables of Actigraph; e) D-PPAC final items of factor 2; f) C-PPAC final items of factor 2. 
TABLE 4 Internal consistency and test-retest reliability of the daily and clinical visit versions of PROactive Physical Activity in COPD (chronic obstructive pulmonary disease) instruments (D-PPAC and C-PPAC, respectively)

\begin{tabular}{|c|c|c|c|c|c|c|c|c|c|c|}
\hline & \multicolumn{2}{|c|}{ Cronbach's $\alpha$} & \multicolumn{8}{|c|}{$\operatorname{ICC} \S$} \\
\hline & Examination $1^{\#}$ & Examination $2^{\pi}$ & Weekly $^{+}$ & Day 1 & Day 2 & Day 3 & Day 4 & Day 5 & Day 6 & Day 7 \\
\hline \multicolumn{11}{|l|}{ D-PPAC } \\
\hline Factor 1: amount with Actigraph & 0.860 & 0.839 & 0.93 & 0.81 & 0.81 & 0.82 & 0.73 & 0.71 & 0.87 & 0.87 \\
\hline Factor 2: difficulty & 0.944 & 0.951 & 0.93 & 0.71 & 0.84 & 0.85 & 0.89 & 0.79 & 0.84 & 0.85 \\
\hline \multicolumn{11}{|l|}{ C-PPAC } \\
\hline Factor 2: difficulty & 0.927 & 0.940 & 0.90 & & & & & & & \\
\hline
\end{tabular}

ICC: intraclass correlation coefficient. ": For D-PPAC the mean value for the first 7 days of daily assessments (week 1 for arm 1/week 5 for arm 2) and for C-PPAC the value for the first assessment (visit 3 for arm 1/visit 1 for arm 2); ${ }^{1}$ : for D-PPAC the mean for the second 7 days of daily assessments (week 2 for arm 1/week 6 for arm 2) and for C-PPAC the value for the second assessment (visit 4 for arm 1/visit 2 for arm $2)_{;}{ }^{+}$: comparison of mean values from week 1 with mean values from week 2 for D-PPAC, and comparison of first and second administration for each patient (week 1 and week 2 in arm 1, and week 3 and week 4 in arm 2) for C-PPAC; ${ }^{\S}$ : for patients randomised to arm 1, the score from day 1 in week 1 was compared to the score from day 1 in week 2, the score from day 2 in week 1 was compared to the score from day 2 in week 2, and so on. For patients randomised to arm 2, the score from day 1 in week 5 was compared to the score from day 1 in week 6 , the score from day 2 in week 5 was compared to the score from day 2 in week 6 , and so on.

correlations with health-related quality of life, dyspnoea and exercise capacity (table 5). Known-groups validity was good in both instruments, with PPAC scores differentiating across grades of dyspnoea, stable from exacerbated patients at baseline and tertiles of physical activity levels (using variables not included in the PPAC scoring, such as intensity) (online supplementary table E9). All correlations with unrelated constructs such as height, socioeconomic status and baseline heart rate were weak; supporting the discriminant validity of both PPAC instruments (online supplementary table E10).

\section{Discussion}

Our analyses resulted in two innovative reliable and valid instruments to fully evaluate the concept of physical activity in patients with COPD: the daily (D-PPAC) and the clinical visit (C-PPAC) versions of the PROactive instruments. The PPAC instruments are hybrid tools that combine PRO and activity monitors to assess physical activity. Interestingly, the C-PPAC turned out to have nearly twice as many items as the D-PPAC. We are not surprised by this fact, because some activities are not performed every day although they are covered in a 1-week period, therefore data about difficulties with such activities may not be captured with a 1-day-recall item but are captured with a 1-week-recall item.

The first important finding of the study confirms that the concept "physical activity" in patients with COPD has two domains, which we labelled "amount of physical activity" and "difficulty with physical activity". The

TABLE 5 Correlation of daily and clinical visit versions of PROactive Physical Activity in COPD (chronic obstructive pulmonary disease) instruments (D-PPAC and C-PPAC, respectively) with health status, dyspnoea and exercise capacity (convergent validity)

\begin{tabular}{|c|c|c|c|c|c|c|}
\hline & CRQ dyspnoea & CRQ fatigue & CCQ total & CAT & mMRC & 6MWD \\
\hline \multicolumn{7}{|l|}{ D-PPAC } \\
\hline Factor 1: amount with Dynaport & $0.36 *$ & $0.18^{*}$ & $-0.20 *$ & $-0.23^{*}$ & $-0.42^{*}$ & $0.55^{*}$ \\
\hline Factor 1: amount with Actigraph & $0.34^{*}$ & $0.17 *$ & $-0.19 *$ & $-0.20 *$ & $-0.41 *$ & $0.55^{*}$ \\
\hline Factor 2: difficulty & $0.74^{*}$ & $0.66^{*}$ & $-0.72^{*}$ & $-0.66^{*}$ & $-0.56 *$ & $0.41 *$ \\
\hline \multicolumn{7}{|l|}{ C-PPAC } \\
\hline Factor 1: amount with Dynaport & $0.36 *$ & $0.37^{*}$ & $-0.37^{*}$ & $-0.38 *$ & $-0.53^{*}$ & $0.62^{*}$ \\
\hline Factor 1: amount with Actigraph & $0.35^{*}$ & $0.36 *$ & $-0.34^{*}$ & $-0.37^{*}$ & $-0.55^{*}$ & $0.65^{*}$ \\
\hline Factor 2: difficulty & $0.86^{*}$ & $0.73^{*}$ & $-0.85^{*}$ & $-0.82^{*}$ & $-0.70 *$ & $0.49 *$ \\
\hline
\end{tabular}

Bold type represents the observed correlations agreed with the expected correlations set by a panel of experts. CRQ: chronic respiratory questionnaire; CCQ: clinical COPD questionnaire; CAT: COPD assessment test; mMRC: modified Medical Research Council dyspnoea scale; 6MWD: 6-min walking distance. *: $p<0.05$. 
"amount" domain is covered by two PRO items (amount of walking outside and chores outside) and by two variables from the activity monitor (steps and VMU), in both PPAC instruments. The inclusion of other items or activity monitor outputs did not enrich the final score. The combination of PRO and activity monitor data yields a much more robust measure of "amount" than either of the components, a unique observation from our study. The "difficulty" domain contains only PRO items and includes items about avoiding or pacing activities and breathlessness during activities. Demonstrating that physical activity is structured in two domains concurs with our qualitative research of experience of physical activity where COPD patients universally described three themes: "amount of", "symptoms during" and "adaptations to" [11]. Based on the analysis of this study, the themes "symptoms" and "adaptations" merged into a single domain ("difficulty").

The second relevant finding is that the final PPAC instruments are hybrid tools combining PRO and activity monitors for the assessment of amount of physical activity in COPD patients. Our comprehensive analyses clearly showed that neither PRO nor activity monitors alone could discriminate well $($ PSI $<0.4)$ within the latent patient-centred construct "amount of physical activity", while the combination of both tools achieved good discrimination at all ranges of the scale. Restricting assessment to either a PRO or an activity monitor does not provide a complete measure of the amount of physical activity. It is possible that activity monitors do not totally capture activities of low intensity, or that relevant activity is not appropriately recalled by patients.

It could be argued that the combination of the PRO with the accelerometer may impair the use of the PPAC instruments. However, the use of activity monitors is becoming more frequent both in clinical practice [10] and in clinical trials [28-30]. In addition, research shows limitations in using only questionnaires for assessing physical activity [13]. The patient-centred development of the PPAC instruments provides comprehensive assessment of physical activity incorporating the patient viewpoint. Importantly, the PPAC instruments will not be limited to the two activity monitors reported in this paper: other activity monitors can be combined with the PRO items after appropriate validation is complete.

It could be argued that some of the excluded items should have been kept in the PPAC instruments due to their clinical relevance. For example, in the "amount" domain, no items about activities inside the house were kept. The item "chores inside the house" did not fit well with the rest of the items according to exploratory factor analysis and showed differential item functioning between males and females (online supplementary table E5). In addition, some male patients did not understand the item during interviews or cognitive debriefings because they considered this item to be more relevant to females. Two items about outside activities were included ("walking outside" and "chores outside"). Although superficially similar, these two items increased discrimination and covered different parts of the range of physical activity, as shown in PSI values and person-item maps. Interestingly, they completely overlapped with the range covered by "chores inside". Finally, VMU, a variable from the activity monitors, showed the best discriminant ability at low amounts of activity (online supplementary fig. E1), suggesting that it conceptually covers what the item "chores inside" was not able to capture.

The item "difficulty climbing stairs" could seem to be important because climbing stairs represents a substantial effort, and was also identified by patients as a priority during interviews and focus groups [11]. However, Rasch analysis showed improper ordering of the item, suggested a poor fit with the other items, presented a floor effect and showed high correlation with other remaining items in the difficulty domain (online supplementary table E5). Both "chores inside the house" and "difficulty climbing stairs" are examples of items that are intuitively relevant which were removed based on corroborating arguments from different sources (statistics and qualitative studies).

Both PPAC instruments demonstrated construct validity, showing ability to measure the construct that they intended to measure. As expected, "amount" scores exhibited weak correlations with health status, and moderate correlations with dyspnoea and exercise capacity, while "difficulty" scores showed moderate-to-strong correlations with health status, dyspnoea and exercise capacity. Additionally, all correlations were $<0.9$, demonstrating that the PPAC instruments provide different information than existing scales, i.e. that PPAC instruments indeed measure physical activity and not exercise capacity or health status [23]. Similarly, the instruments showed good known-groups validity when comparing across previously defined groups, detecting differences in physical activity (using variables not included in the PPAC scoring) between them. Finally, the discriminant validity showed that the difficulty and amount domains from the PPAC instruments were unrelated (predefined as correlation $<0.3$ ) to the constructs that were expected to be unrelated such as patient height. In comparison with previous studies that aimed to validate questionnaires to measure physical activity in patients with COPD, our instruments exhibited better correlations for convergent validity. For example, correlations of $\sim 0.37$ were found between the several outputs from the Yale Physical Activity Survey and the 6-min walking distance [31], compared to correlations of $\sim 0.55$ in our instruments. 
Measuring the experience of physical activity is important for COPD management because physical activity is important from the patient's perspective [11] and thus for their daily life, and no valid instruments are currently available to capture it. In particular, because patients report that treatments (which need not be limited to pharmacological approaches) that improve physical activity are of value to them [11], the availability of PPAC tools which have been developed in line with current guidance will help future clinical trials aiming to include physical activity as an regulatory-accepted outcome [14].

A strength of this study is that the instruments were developed according to current best practice [14, 17], and their psychometric properties were rigorously tested using appropriate methodology and predefined and rigorous statistical procedures [32]. Both the D-PPAC and the C-PPAC item pools were developed through a systematic process of literature reviews [12, 13], input from experts and, as recommended [33-35], input from patients through qualitative research using one-to-one interviews, focus groups and cognitive debriefing interviews. Patients recruited were representative of a wide spectrum of COPD disease severity, comorbidities and different geographical and cultural backgrounds [11]. The information from this qualitative research was also used during the item reduction process when deciding to remove or keep an item. An advisory board provided advice to guide the whole process for the item reduction and validation of the tools. Moreover, the item reduction and initial validation reported here were also based on a heterogeneous sample of patients with COPD that covered a whole range of physical activity and other clinical determinants.

Another strength of the PPAC instruments is that they are based on a conceptual framework. A systematic review identified the absence of a conceptual framework of physical activity in all existing instruments to assess it in patients with COPD [13], which raises doubts about the validity of these instruments. We drafted an initial conceptual framework from extensive patient input and literature review [11], which was refined after item reduction and confirmed using appropriate statistical methods.

Our study has some limitations. Firstly, although the sample size was sufficient and the population was quite heterogeneous to achieve the objective of the study, further validation is needed in specific subgroups of patients with COPD. For example, the person item maps of the "difficulty" domain (factor 2) suggest that our instruments perhaps cover patients with greater difficulty in physical activity than the difficulty shown by patients in our sample, since the person distribution is skewed to the lower end of the scale. Secondly, the PPAC instruments have been validated only in a European population and using only three different languages. Further studies are ongoing to validate the PPAC instruments in patients with both milder and more severe COPD, from other countries and using validated translations of the instruments. In the same way, additional studies are required to confirm the best scoring system for the PPAC instruments, to assess responsiveness to change due to interventions and to define levels of response.

In conclusion, both the D-PPAC and the C-PPAC, combining a short list of PRO items and two activity monitor variables, provide a simple, valid and reliable measure of physical activity in COPD patients. These tools will be further evaluated in different clinical situations and for responsiveness to drug and nondrug interventions.

\section{Acknowledgements}

The PROactive Consortium members are as follows. Nathalie Ivanoff: Almirall, Barcelona, Spain; Niklas Karlsson and Solange Corriol-Rohou: AstraZeneca AB, Mölndal, Sweden; Ian Jarrod: British Lung Foundation, London, UK; Damijen Erzen: Boehringer Ingelheim, Nieder-Ingelheim, Germany; Caterina Brindicci, Tim Higenbottam and Mario Scuri: Chiesi Farmaceutici S.A. Parma, Italy; Paul McBride: Choice Healthcare Solutions, Hitchin, UK; Nadia Kamel: European Respiratory Society, Lausanne, Switzerland; Margaret Tabberer: GlaxoSmithKline, Uxbridge, UK; Thierry Troosters and Fabienne Dobbels: Katholieke Universiteit Leuven, Leuven, Belgium; Judith Garcia-Aymerich: Municipal Institute of Medical Research, Barcelona, Spain; Pim de Boer: Netherlands Asthma Foundation, Amersfoort, The Netherlands; Karoly Kulich and Alastair Glendenning: Novartis, Basel, Switzerland; Katja Rudell and Frederick J. Wilson: Pfizer Walton Oaks, UK; Michael I. Polkey and Nick S. Hopkinson: Royal Brompton and Harefield NHS Foundation Trust, London, UK; Ioannis Vogiatzis: Thorax Research Foundation, Athens, Greece; Enkeleida Nikai: UCB, Brussels, Belgium; Thys van der Molen and Corina De Jong: University Medical Center, Groningen, The Netherlands; Roberto A. Rabinovich and Bill MacNee: University of Edinburgh, Edinburgh, UK; Milo A. Puhan and Anja Frei: University of Zurich, Zurich, Switzerland.

We are grateful for the input from the PROactive project ethics board, advisory board and patient input platform (www. proactivecopd.com/about/advisory-boards).

\section{References}

1 Nelson ME, Rejeski WJ, Blair SN, et al. Physical activity and public health in older adults: recommendation from the American College of Sports Medicine and the American Heart Association. Med Sci Sports Exerc 2007; 39: 1435-1445.

2 Garcia-Aymerich J, Lange P, Benet M, et al. Regular physical activity reduces hospital admission and mortality in chronic obstructive pulmonary disease: a population based cohort study. Thorax 2006; 61: 772-778. 
Garcia-Aymerich J, Lange P, Serra I, et al. Time-dependent confounding in the study of the effects of regular physical activity in chronic obstructive pulmonary disease: an application of the marginal structural model. Ann Epidemiol 2008; 18: 775-783.

4 Vorrink SNW, Kort HSM, Troosters T, et al. Level of daily physical activity in individuals with COPD compared with healthy controls. Respir Res 2011; 12: 33-40.

5 Shrikrishna D, Patel M, Tanner RJ, et al. Quadriceps wasting and physical inactivity in patients with COPD. Eur Respir J 2012; 40: 1115-1122.

6 Troosters T, Sciurba F, Battaglia S, et al. Physical inactivity in patients with COPD, a controlled multi-center pilot-study. Respir Med 2010; 104: 1005-1011.

7 Pitta F, Troosters T, Spruit M, et al. Characteristics of physical activities in daily life in chronic obstructive pulmonary disease. Am J Respir Crit Care Med 2005; 171: 972-977.

8 Giacomini M, DeJean D, Simeonov D, et al. Experiences of living and dying with COPD: a systematic review and synthesis of the qualitative empirical literature. Ont Health Technol Assess Ser 2012; 12: 1-47.

9 Gimeno-Santos E, Frei A, Steurer-Stey C, et al. Determinants and outcomes of physical activity in patients with COPD: a systematic review. Thorax 2014; 69: 731-739.

10 Van Remoortel H, Giavedoni S, Raste Y, et al. Validity of activity monitors in health and chronic disease: a systematic review. Int J Behav Nutr Phys Act 2012; 84-106.

11 Dobbels F, de Jong C, Drost E, et al. The PROactive innovative conceptual framework on physical activity. Eur Respir J 2014; 44: 1223-1233.

12 Williams K, Frei A, Vetsch A, et al. Patient-reported physical activity questionnaires: a systematic review of content and format. Health Qual Life Outcomes 2012; 10: 28-45.

13 Gimeno-Santos E, Frei A, Dobbels F, et al. Validity of instruments to measure physical activity may be questionable due to a lack of conceptual frameworks: a systematic review. Health Qual Life Outcomes 2011; 9: 86-99.

14 US Department of Health and Human Services Food and Drug Administration. Guidance for Industry. Patient-Reported Outcomes Measures: Use in Medical Product Development to Support Labeling Claims. 2009. www.fda.gov/downloads/Drugs/GuidanceComplianceRegulatoryInformation/Guidances/UCM193282.pdf Date last accessed: July 26, 2014.

15 Rabinovich RA, Louvaris Z, Raste Y, et al. Validity of physical activity monitors during daily life in patients with COPD. Eur Respir J 2013; 42: 1205-1215.

16 Van Remoortel H, Raste Y, Louvaris Z, et al. Validity of six activity monitors in chronic obstructive pulmonary disease: a comparison with indirect calorimetry. PLoS One 2012; 7: e39198.

17 Committee for Medicinal Products for Human Use, European Medicines Agency. Qualification of Novel Methodologies for Drug Development: Guidance to Applicants. 2008. www.ema.europa.eu/docs/en_GB/document_ library/Regulatory_and_procedural_guideline/2009/10/WC500004201.pdf Date last accessed: July 26, 2014.

18 Jones PW, Harding G, Berry P, et al. Development and first validation of the COPD Assessment Test. Eur Respir J 2009; 34: 648-654.

19 Jones PW, Chen W-H, Wilcox TK, et al. Characterizing and quantifying the symptomatic features of COPD exacerbations. Chest 2011; 139: 1388-1394.

20 Reeve BB, Hays RD, Bjorner JB, et al. Psychometric evaluation and calibration of health-related quality of life item banks: plans for the Patient-Reported Outcomes Measurement Information System (PROMIS). Med Care 2007; 45: S22-S31.

21 Andrich D. A rating formulation for ordered response categories. Psychometrika 1978; 43: 561-573.

22 Meguro M, Barley EA, Spencer S, et al. Development and validation of an improved, COPD-specific version of the St. George Respiratory Questionnaire. Chest 2007; 132: 456-463.

23 Pesudovs K, Burr JM, Harley C, et al. The development, assessment, and selection of questionnaires. Optom Vis Sci 2007; 84: 663-674.

24 Crane PK, Gibbons LE, Jolley L, et al. Differential item functioning analysis with ordinal logistic regression techniques. DIFdetect and difwithpar. Med Care 2006; 44: S115-S123.

25 Choi SW, Gibbons LE, Crane PK. lordif: an R package for detecting differential item functioning using iterative hybrid ordinal logistic regression/item response theory and Monte Carlo simulations. J Stat Softw 2011; 39: 1-30.

26 Hill CD, Edwards MC, Thissen D, et al. Practical issues in the application of item response theory: a demonstration using items from the pediatric quality of life (PedsQL) 4.0 generic core scales. Med Care 2007; 45: S39-S47.

27 Altman DG, Bland JM. Measurement in medicine: the analysis of method comparison studies. Statistician 1983; 32: 307-317.

28 Troosters T, Sciurba FC, Decramer M, et al. Tiotropium in patients with moderate COPD naive to maintenance therapy: a randomised placebo-controlled trial. NPJ Prim Care Respir Med 2014; 24: 14003.

29 Hataji $\mathrm{O}$, Naito M, Ito K, et al. Indacaterol improves daily physical activity in patients with chronic obstructive pulmonary disease. Int J Chron Obstruct Pulmon Dis 2013; 8: 1-5.

30 O'Donnell DE, Casaburi R, Vincken W, et al. Effect of indacaterol on exercise endurance and lung hyperinflation in COPD. Respir Med 2011; 105: 1030-1036.

31 Donaire-Gonzalez D, Gimeno-Santos E, Serra I, et al. Validación del cuestionario de actividad física de Yale en pacientes con enfermedad pulmonar obstructiva crónica. [Validation of the Yale Physical Activity Survey in chronic obstructive pulmonary disease patients.] Arch Bronconeumol 2011; 47: 552-560.

32 Frost $\mathrm{MH}$, Reeve BB, Liepa AM, et al. What is sufficient evidence for the reliability and validity of patient-reported outcome measures? Value Health 2007; 10: Suppl. 2, S94-S105.

33 Snyder CF, Watson ME, Jackson JD, et al. Patient-reported outcome instrument selection: designing a measurement strategy. Value Health 2007; 10: Suppl. 2, S76-S85.

34 Turner RR, Quittner AL, Parasuraman BM, et al. Patient-reported outcomes: instrument development and selection issues. Value Health 2007; 10: Suppl. 2, S86-S93.

35 Patrick DL, Burke LB, Powers JH, et al. Patient-reported outcomes to support medical product labeling claims: FDA perspective. Value Health 2007; 10: Suppl. 2, S125-S137. 